\title{
Single Polymer Chains as Specific Transducers of Molecular Recognition in Scanning Probe
}

\author{
Microscopy
}

\author{
Rachel Gabai, Lior Segev and Ernesto Joselevich*
}

Department of Materials and Interfaces, Weizmann Institute of Science, Rehovot 76100, Israel.

Email: ernesto.joselevich@weizmann.ac.il

\section{Supporting Information}

\section{Characterization and selection of single-molecule entropic probes}

The probes, consisting of biotin-PEG-functionalized AFM tips, were first characterized by conventional force spectroscopy on a substrate containing covalently immobilized streptavidin molecules, and then selected according to their characteristics. Figure S1 shows typical force-distance curves recorded with three different types of probes, designated as A, B and C. Figure S1a displays a typical force-distance curve for a "type A" probe. Here, a single peak is observed, corresponding to the elastic behavior of the 
tether, up to ligand-receptor unbinding force. The latter was consistent with previously reported unbinding forces for biotin-streptavidin at the fast loading-rate limit, $200 \mathrm{pN}$. The observed elastic behavior of the tether is consistent with that of PEG in water, and can be well fitted to eq. 4 . The detachment or "pull-off" takes place at a distance of $\sim 30$ $\mathrm{nm}$ from the tip-surface contact position, corresponding to the contour length of the PEG tether, $30 \pm 5 \mathrm{~nm}$. This means that in this type of probes there is only one tethered ligand molecule capable of reaching the surface, and it is attached to the very apex of the AFM tip. Figure S1b displays a typical force-distance curve for a "type B" probe. Here, there is also a single peak, but located at a distance from contact significantly shorter than $30 \mathrm{~nm}$. These probes also contain a single tethered ligand molecule capable of reaching the surface, however it is not attached to the apex of the AFM tip, but several nanometers off the apex toward the base of the pyramid. Figure S1c displays a typical force-distance curve for a "type C" probe, showing multiple peaks at different distances from contact, up to $\sim 30 \mathrm{~nm}$, often simultaneously, and always reproducibly at the same distances. These probes contain multiple tethered ligand molecules capable of reaching the surface. "Type D" probes (not shown) display no force-distance peaks because they do not contain any tethered ligand capable of reaching the surface.

For most of the experiments described herein, only probes containing a single tethered ligand at the apex (type "A") were selected. For certain control experiments (vide infra), mentioned later, type $\mathrm{C}$ probes were selected. 


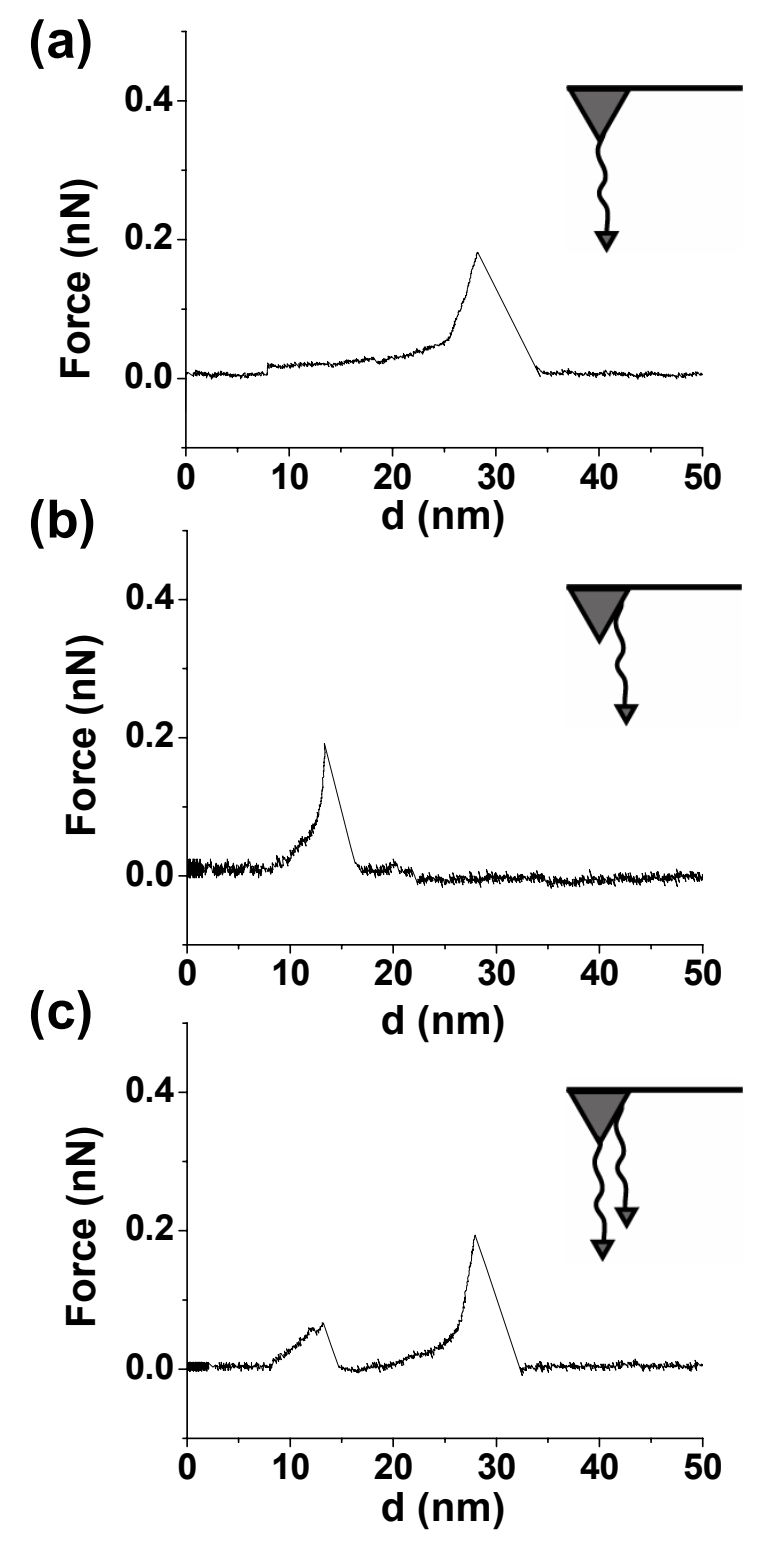

Figure S1. Probe characterization and selection by force spectroscopy. (a) Probe containing a single tethered ligand at the apex of the AFM tip. (b) Probe containing one tethered ligand but not at the tip apex. (c) Probe containing multiple PEG-biotin molecules.

\section{Experiments with multiple binding probes}


Additional evidence for the proposed mechanism of specific probing of molecular recogntion is obtained when this experiment is done with probes having several tethered biotin molecules at different positions ("type C", as the one in Figure S1c), as shown in Figure S2. Then, the second-harmonic amplitude images show a multiple-tip artifact at low peak-to-peak amplitude, but the multiplet features turn into sharp single features as the peak-to-peak amplitude increases. This is attributed to the fact that at low amplitude two ligands can bind the receptor molecule on the surface, but when the amplitude approaches the contour length, only the one ligand tethered to the apex of the tip can bind the receptor.

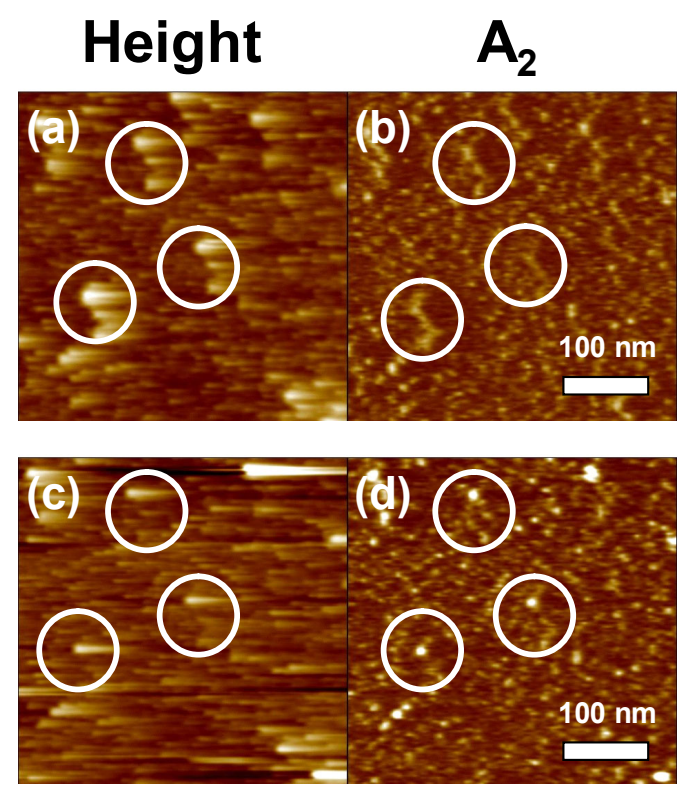

Figure S2. Effect of amplitude on the molecular recognition imaging of streptavidin by tip functionalized with multiple biotin-PEG groups ("type C"). (a) Topography and (b) second-harmonic amplitude image at peak-to-peak amplitude $A=10 \mathrm{~nm}$. (c) Topography and (d) second-harmonic amplitude image at peak-to-peak amplitude $\mathrm{A}=30 \mathrm{~nm}$. 